\title{
Remote Biosensing with Polychromatic Optical Waveguide Using Blue Light-Emitting Organic Nanowires Hybridized with Quantum Dots
}

\author{
Eun Hei Cho, Bong-Gi Kim, Sumin Jun, Jubok Lee, Dong Hyuk Park, Kwang-Sup Lee, \\ Jeongyong Kim,* Jinsang Kim,* and Jinsoo Joo*
}

Nanometer-scale optical waveguides are attractive due to their potential applicability in photonic integration, optoelectronic communication, and optical sensors. Nanoscale white light-emitting and/or polychromatic optical waveguides are desired for miniature white-light generators in microphotonic circuits. Here, polychromatic (i.e., blue, green, and red) optical waveguiding characteristics are presented using a novel hybrid composite of highly crystalline blue light-emitting organic nanowires (NWs) combined with blue, green, and red $\mathrm{CdSe} / \mathrm{ZnS}$ quantum dots (QDs). Near white-color waveguiding is achieved for organic NWs hybridized with green and red QDs. Light, emitted from QDs, can be transferred to the organic NW and then optically waveguided through highly packed $\pi$-conjugated organic molecules in the NW with different decay characteristics. Remote biosensing using dye-attached biomaterials is presented by adapting the transportation of QD-emitted light through the organic NW. for applications in photonic integration, optoelectronic communication, and miniaturized optical sensors. ${ }^{[7,8]}$ Propagation of photons and surface plasmon polaritons is the major waveguiding mechanism responsible for lowdimensional semiconductors and metal nanostructures, respectively. In organic semiconducting waveguide materials, exciton polaritons are formed due to a strong coupling between Frenkel excitons and photon-phonon transverse wave (i.e., polaritons), ${ }^{\left[{ }^{[]}\right.}$and possess excellent propagation properties compared with the uncoupled waveguide modes. ${ }^{[10]}$ Crystalline 1D organic nanostructures composed of highly ordered $\pi$-conjugated molecules can generate more excitons

\section{Introduction}

Nanometer-scale, 1D optical waveguides, fabricated using inorganic semiconductors, ${ }^{[1,2]}$ metals, ${ }^{[3,4]}$ polymers, ${ }^{[5]}$ and organic small molecules, ${ }^{[6]}$ have been widely investigated upon excitation, and are characterized by efficient propagation of polaritons, good environmental stability, and anisotropic energy/charge transport properties, thereby possibly rendering enhanced photonic performance useful for miniaturized devices. ${ }^{[11]}$
E. H. Cho, Prof. J. Joo

Department of Physics

Korea University

Seoul 136-713, South Korea

E-mail: jjoo@korea.ac.kr

Dr. B.-G. Kim, Prof. J. Kim

Materials Science and Engineering

Macromolecular Science and Engineering

and Chemical Engineering

University of Michigan

MI 48109, USA

E-mail: jinsang@umich.edu

S. Jun, Prof. K.-S. Lee

Department of Advanced Materials

Hannam University

Daejeon 305-811, South Korea

J. Lee, Prof. J. Kim

IBS Center for Integrated Nanostructure Physics (CINAP)

Institute for Basic Science (IBS)

Department of Energy Science

Sungkyunkwan University

Suwon 440-746, South Korea

E-mail: j.kim@skku.edu
Prof. D. H. Park

Materials Science and Engineering

University of Michigan

MI 48109, USA

Prof. D. H. Park

Division of Nano-systems Engineering

Department of Advanced Fiber Engineering

Inha University

Incheon 402-751, South Korea

Prof. D. H. Park

Hybrid Materials for Sustainability

Department of Materials Science and Engineering

Seoul National University

Seoul 151-742, South Korea

DOI: 10.1002/adfm.201304039 
(a)

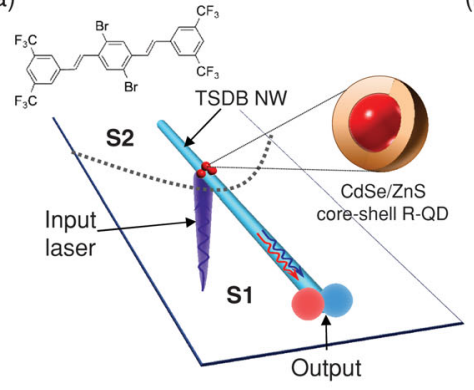

(b)

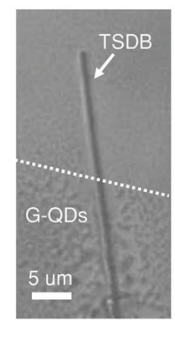

(c)

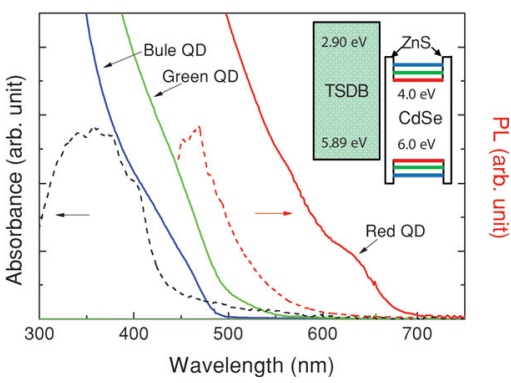

Figure 1. (a) Schematic chemical structure of TSDB molecule (top). Schematic illustration of the polychromatic optical waveguiding experiment using a TSDB NW/QDs hybrid system. (b) Microscope image of a TSDB/G-QD (green light-emitting QD) hybrid NW. The lower half surface was coated with QDs. (c) UV-vis absorption spectra of TSDB NWs (black dotted curve) and QDs (solid curves) in methanol solution. PL spectrum of TSDB NWs (red dotted curve). Inset: Schematic energy levels of TSDB/QD hybrid NW.

A better confinement of optical signals within an optical waveguide, enabling efficient transfer of propagating modes, has been shown to require a high refractive index $n$. Although semiconducting organic materials bear a great promise to be used in future nanosize devices on flexible substrate, organic molecular crystals are generally characterized by low refractive indices $(\sim 1.5)$, which causes optical propagation loss and a relatively strong substrate effect. Accordingly, a great effort has been made to increase the refractive indices of organic crystals as well as to minimize the defects and grain boundaries. Along these lines, we designed and synthesized a $\pi$-conjugated semiconducting organic molecule, 1,4-bis(3,5-bis(trifluoro methyl) styryl)-2,5-dibromobenzene (TSDB), containing halogen atoms ( $\mathrm{F}$ and $\mathrm{Br}$ ) for this study. Incorporation of halogen atoms in an organic molecule increases the refractive index by enhancing the electron density of the molecule by means of a heavy atom effect. In addition, fluoride can induce closer packing of organic molecules through hydrophobic effect. Moreover, a suitable application of $\mathrm{C}-\mathrm{F}$ bonds can contribute to a low absorption loss at longer wavelengths because the fluorinated macromolecules have good propagation characteristics owing to the improved optical transparency in the near infrared region at $1.3 \mu \mathrm{m}$ and $1.55 \mu \mathrm{m} .{ }^{[12]}$ The $\pi$-conjugated backbone also enriches the TSDB with $\pi$-electron, which contributes to the enhancement of refractive index. Therefore, a combination of the molecular design components of TSDB molecule resulted in anisotropically self-assembled and well-aligned $\pi-\pi$ stacked organic nanowires (NWs) with a relatively high refractive index of $1.71-1.77$.

Herein, we report unique polychromatic optical waveguide with low absorption loss through a novel hybrid composite of highly crystalline blue light-emitting organic TSDB NWs combined with blue, green, and red CdSe/ZnS quantum dots (QDs). The output photoluminescence (PL) signals through the NWs could be manipulated to range from $470 \mathrm{~nm}$ to $650 \mathrm{~nm}$ simply by controlling the core diameter of the secondary lightemitting CdSe/ZnS QDs. Near-white PL waveguiding was achieved by coupling green and red QDs on the surface of a single TSDB NW. The propagation loss of the PL intensity as a function of the propagation distance was calculated to investigate the mechanism of optical waveguiding in this system. Optical signals propagated by means of a strong coupling of excitons and polaritons through tightly packed $\pi-\pi$ stacking of TSDB molecules in hybrid NWs with QDs. To demonstrate the applicability of such hybrid system, remote bio-material sensing based on the optical waveguiding characteristics of the TSDB NW and QDs light emission was designed and successfully performed through the enhanced detection of a signal from dye attached bio-materials.

\section{Results and Discussion}

\subsection{Fabrication of TSDB NWs and CdSe/ZnS QDs and Optical Characteristics}

Figure 1a shows the chemical structure of a TSDB molecule and the schematic illustration of the optical waveguiding experiment that uses the TSDB/QDs hybrid nanosystems. The TSDB molecules were self-assembled in the form of NWs whose axial length was tens of microns. The width and height of the TSDB NWs were about $400 \mathrm{~nm}$ and $200 \mathrm{~nm}$, respectively. The core-shell CdSe/ZnS QDs were passivated with 11-mercapto1-undecanol functionalized terminal, which assists in the homogeneous dispersion of QDs in methanol and in their easy attachment to the NWs. The NWs were placed on a cover glass substrate and half of the NW's length was coated with QDs by drop casting in order to study waveguiding characteristics of locally generated emission of light from QDs. Consistent with our design, optical microscope images of the samples revealed that one half of the TSDB NWs were exposed to the atmosphere and the remaining part was coated with the QDs (Figure 1b). The attachment of QDs to the surface of a NW was also confirmed by three-dimensional atomic force microscope (AFM) imaging (Figure S1). The surface of a pristine TSDB NW without the QDs was relatively flat, while the other half of the TSDB NW's length, coated with the QDs, was characterized by an uneven surface due to the size of the QDs (Figure 1b and Figure S1). The thickness of a QD layer on a NW was estimated to be $25.5 \pm 5.6 \mathrm{~nm}$ (Figure $\mathrm{S} 1$ ).

Figure 1c shows the optical properties of TSDB NWs and CdSe/ZnS QDs. UV-vis absorption and PL spectra of TSDB NWs on a cover glass substrate are shown as dotted lines. Solution absorption spectra of three types of QDs (solid curves) exhibited different absorption edges; the central PL peaks of blue QDs at $\lambda_{\mathrm{em}}=472 \mathrm{~nm}$, green QDs at $\lambda_{\mathrm{em}}=532 \mathrm{~nm}$, and red 
QDs at $\lambda_{\mathrm{em}}=635 \mathrm{~nm}$. The TSDB NWs showed blue emission with a PL peak at $468 \mathrm{~nm}$, which corresponds to the $\pi-\pi *$ transition, as well as vibrational shoulder peaks at $480 \mathrm{~nm}$ and $491 \mathrm{~nm}$ (Figure 1c). The main central peak and the shoulder peak of the TSDB NW absorption spectrum are at $375 \mathrm{~nm}$ and $400 \mathrm{~nm}$, respectively. Above $600 \mathrm{~nm}$ almost no absorption is observed, implying no absorption loss at long wavelengths. Schematic energy levels of TSDB molecules and QDs are shown in the inset of Figure 1c. The optical characteristics imply that emission and re-absorption of light from TSDB molecules to QDs is possible.

\subsection{Optical Waveguiding Characteristics of Pristine TSDB NWs}

Nanoscale optical waveguiding characteristics of a pristine single NW were investigated by means of the output PL spectra using a high-resolution ( $\leq 200 \mathrm{~nm}$ ) laser confocal microscope (LCM) system equipped with a diode laser $\left(\lambda_{\mathrm{ex}}=405 \mathrm{~nm}\right)$. We moved the excitation point by focused input laser along the NW's axial direction while the position of detection point (output) was fixed at the end of the NW, as shown in Figure 2a. Using the LCM system, both the excitation and the detection points were placed normally to the NW (Figure 2a (top image)), which is relatively simple to implement in optical waveguiding not requiring the fiber taper. ${ }^{[13]}$ In this configuration, the excitation laser can not be directly coupled into the guided mode of the NW due to the high refractive index of the TSDB NW. This makes sure that the propagation signal along the NW is only the fluorescence of NW originated at the excitation location. In Figure $2 \mathrm{a}$, the output blue light emission was observed at both ends of the TSDB NW, implying that it can easily propagate through the NW. The waveguided PL intensity $I(x)$ decreased as the propagation distance $x$ increased (Figure $2 \mathrm{~b}$ (left)). We used the intensity equation, $I(x)=I_{0} \exp (-\alpha x)$, to fit the data in Figure $2 \mathrm{~b}$ and estimated the PL decay constant $\alpha$ as $0.0227 \mu^{-1}$, which is much smaller (a much better waveguiding property) than that of other organic crystalline materials with similar dimensions, such as rubrene nanostructures ${ }^{[14]}$ and 1,5-diaminoanthraquinone NWs $\left(\alpha=0.348 \mathrm{\mu m}^{-1}\right.$, even for a freestanding mode with an air cladding). ${ }^{[15]}$ We also observed good waveguiding performance using a few hundred micrometers long TSDB NW with bending structure (see Figure S2).
As shown in Figure 2a (top and bottom images), we designed a different waveguiding experiment using a polydimethylsiloxane (PDMS) microchannel stamp, in order to improve the waveguiding efficiency by changing the substrate. The PDMS stamp on a cover glass has hollow microchannels with a depth and width of about $10 \mu \mathrm{m}$ and $25 \mu \mathrm{m}$, respectively. The TSDB NWs were partially suspended on the convex surface of the PDMS stamp. The excitation laser was moved along the suspended NW's axis with an air background, and the detection point was placed at the end of the suspended NW, as shown in the bottom scheme in Figure 2a. The top image in Figure 2c (right) shows the optical microscope image of the suspended NW on the PDMS stamp. Figure 2c (right) shows the CCD images of the waveguided luminescence obtained from the experiments in which the PDMS microchannel stamp was used. The waveguided PL intensities were stable and decreased weakly as the propagation distance increased (Figure 2c (left)). From the equation $I(x)=I_{0} \exp (-\alpha x)$, the decay constant $\alpha$ was

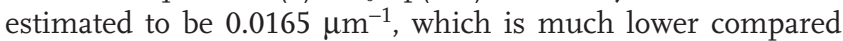
with that of the TSDB NW $\left(\alpha=0.0227 \mu \mathrm{m}^{-1}\right)$ prepared on the cover glass (i.e. using the top scheme in Figure 2a). The output PL peak's shift toward the shorter wavelengths was not observed in the waveguided PL spectra for the suspended TSDB NW, implying that the longer wavelength signal loss of optical waveguiding due to the substrate effect did not appear in this case. These results show that the intrinsic efficiency of pristine TSDB NW is high enough to send its own fluorescence signal to the distance of $\sim 100 \mu \mathrm{m}$.

When a waveguiding NW has a rectangular cross-section with dielectric tensor components $\varepsilon_{\|}$and $\varepsilon_{\perp}$ (along and normal to the NW, respectively), an electromagnetic wave, such as light, can propagate in the waveguide with transverse magnetic (TM) modes. The number of possible propagation waveguiding modes $m$ is given by $m<2 a / \lambda \sqrt{\varepsilon_{\perp}\left(\varepsilon_{\|}-\varepsilon_{s}\right) / \varepsilon_{\|}}$, where $a$ is the width of the waveguide, $\lambda$ is the wavelength, and $\varepsilon_{\mathrm{s}}$ is the dielectric constant of the substrate. ${ }^{[16]}$ When the waveguide has an optically isotropic property, $\varepsilon_{i s o}=\varepsilon_{\perp}=\varepsilon_{\|}$, the cutoff wavelength ( $\left.\lambda_{\text {cutoff }}\right)$ for the waveguide is given by $\lambda_{\text {cutoff }}=2 a \sqrt{\varepsilon_{\text {iso }}-\varepsilon_{s}}$. The $\lambda_{\text {cutoff }}$ of the propagating waveguided light rays could be approximated by using $\varepsilon_{\text {iso }}=n_{\perp}^{2}, \varepsilon_{s}=n_{s}^{2}=1.53^{2}\left(n_{\perp}\right.$ is the refractive index of the waveguide and $n_{\mathrm{s}}$ is the refractive index of a glass substrate). From the dispersion relation, $\lambda_{\text {cutoff }}$ values for the TSDB NW with the width of $a=400 \mathrm{~nm}$ were calculated (a)

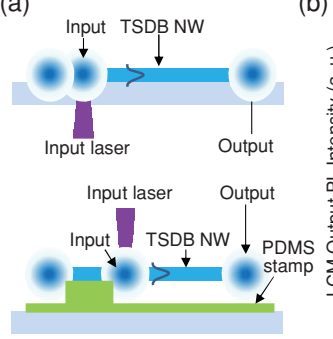

(b)

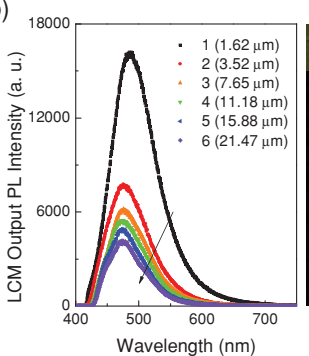

(c)

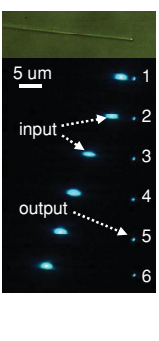

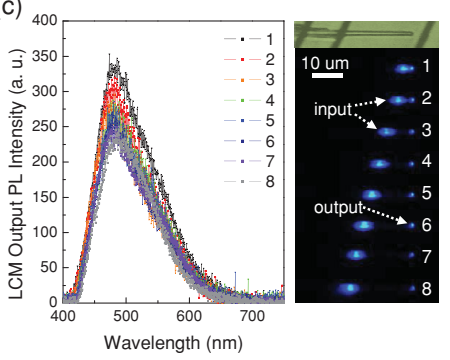

Figure 2. (a) Schematic illustrations of the waveguiding experiments using the NW placed directly on the cover glass (top) and the NW partially suspended on the PDMS stamp (bottom). (b) Output LCM PL spectra (left) and CCD images (right) of the waveguided emissions of a pristine TSDB NW on the cover glass with propagation distances (in the parentheses). The estimated decay constant $\alpha$ was $0.0227 \mu \mathrm{m}^{-1}$ for output PL peak at $490 \mathrm{~nm}$. (c) Output LCM PL spectra (left) and CCD images (right) of the waveguided emissions of a pristine TSDB NW on the PDMS stamp. The estimated

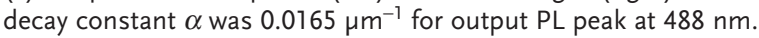


to be 612,656 , and $705 \mathrm{~nm}$ by using the refractive index $(n)$ of the TSDB material $(1.71,1.74$, and 1.77$)$ for the light rays that have wavelengths of 488,532, and $630 \mathrm{~nm}$, respectively. The $n$ values of the NWs were measured by using a spectroscopic ellipsometer (Elli-SE, Ellipso Technology Co.). These estimated $\lambda_{\text {cutoff }}$ values are sufficiently large so that red, green, and blue light emissions from QD can propagate through the TSDB NW as a guiding mode.

\subsection{Polychromatic Optical Waveguiding Characteristics of TSDB/QD Hybrid NWs}

The TSDB NWs were hybridized with a partial attachment of blue, green, and red light-emitting CdSe/ZnS QDs, as shown in Figure 1a. The half of the pristine TSDB NW without QDs is denoted as section 1 (S1), and the remaining part of the QDcoated NW is denoted as section 2 (S2). The detecting position of the light ray that was waveguided through the NW (output PL signal) was fixed perpendicular to the NW axis at the end of the pristine NW. An incident focused laser $\left(\lambda_{\text {ex }}=405 \mathrm{~nm}\right)$ was used as an input optical signal and a few different points of excitation were selected along the axial direction of the NW. For Figure 3, it is noted that the $n$ values of TSDB NWs are 1.71, 1.74, and 1.77 at wavelengths of 488, 532, and $630 \mathrm{~nm}$, respectively.

As shown in Figure 3a, in region S1 the TSDB NWs that were hybridized with the red light-emitting QDs (R-QDs, $\lambda_{\mathrm{em}}=$ $635 \mathrm{~nm}$ ) exhibited emission only from TSDB NWs, and $\lambda_{\max }$ (a)

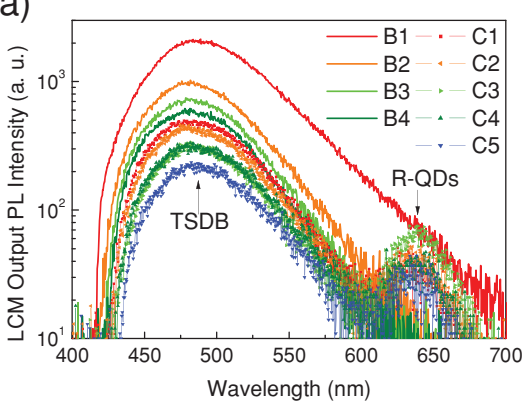

(c)

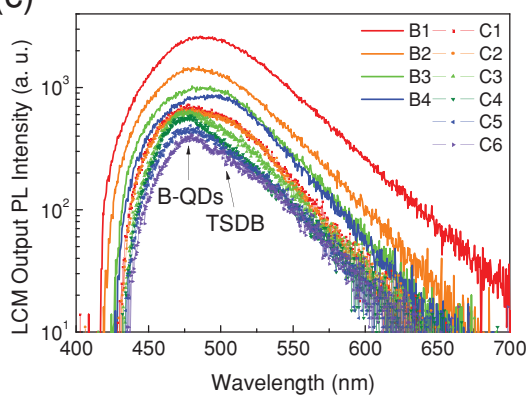

(b)

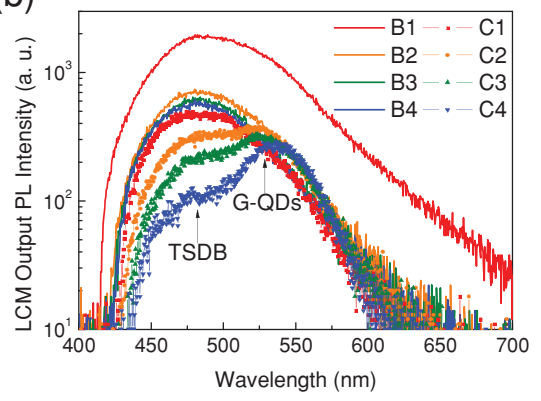

(d)

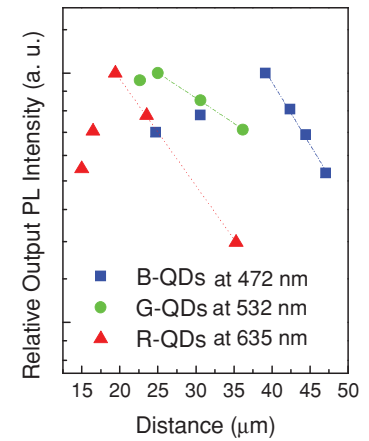

Figure 3. LCM PL spectra of the waveguided emissions (output signals) of (a) a TSDB/R-QD hybrid NW, (b) a TSDB/G-QD hybrid NW, and (c) a TSDB/B-QD hybrid NW. The B1-B4 spectra were obtained from the region S1 of the TSDB NW, as explained in the main text. The output PL spectra with dotted curves ( $\mathrm{Cl}-\mathrm{C} 6$ spectra) were transported from the input laser's point in the TSDB/QD hybrid region S2. (d) Relative output LCM PL intensity of waveguided emissions centered at 472, 532, and $635 \mathrm{~nm}$, as a function of propagation distance corresponding to the blue, green, and red QD emissions, respectively. of the emission exhibited a slight shift toward the blue (from $488 \mathrm{~nm} \mathrm{(B1)} \mathrm{to} 480 \mathrm{~nm}$ (B4)) as the propagation distance increased. In contrast, the PL spectrum in region S2 (C1-C5 spectra in Figure 3a) clearly shows two distinct peaks at $481 \mathrm{~nm}$ and $640 \mathrm{~nm}$, attributable to the TSDB NWs and R-QDs, respectively. While the waveguided output PL intensities of both the TSDB NWs and R-QDs decreased with increasing propagation distance, dual waveguided output signals from TSDB NWs and R-QDs were clearly observed. The same general trend was observed for the TSDB NWs coupled with green light-emitting QDs (G-QDs, $\lambda_{\text {em }}=532 \mathrm{~nm}$ ). The output PL spectra in region S1 (B1-B4 spectra) exhibited only one emission peak, centered at $488 \mathrm{~nm}$, corresponding to the emission from TSDB NWs. The output PL spectra in region S2 (C1-C4 spectra) exhibited two peaks, at $488 \mathrm{~nm}$ and $532 \mathrm{~nm}$, attributable to the TSDB NWs and G-QDs, respectively, as shown in Figure 3b. In general, the PL intensity of the waveguided output decreased for the increasing propagation distance. Interestingly however, the relative intensity inversion between the output PL of G-QDs and that of TSDB NWs was observed when the waveguided light propagated approximately $30 \mu \mathrm{m}$, implying that the emission from G-QDs is characterized by better propagation efficiency compared with that of TSDB NWs. In the same vein, as is shown in Figure 3c, propagation efficiency was different for TSDB NWs coated with the blue light-emitting QDs (B-QDs, $\lambda_{\text {em }}=472 \mathrm{~nm}$ ). Although the intensity of both output PL signals from TSDB NW and B-QDs gradually decreased as the light propagated along the NW, the emission from B-QDs became dominant when the propagation distance was $30.6 \mu \mathrm{m}$. These data demonstrate that the waveguided lights from two different emission sources, TSDB NWs and QDs, can propagate through TSDB/QD hybrid NWs with different decay characteristics through the $\pi-\pi$ molecular interactions and scattering effects.

To analyze the decay characteristics of the waveguided light, the measured output PL spectra (in Figure 3) of the TSDB/QD hybrid NWs were deconvoluted into sub-peaks corresponding to the characteristic wavelengths of $488 \mathrm{~nm}$ for TSDB material, and 472, 532, and $635 \mathrm{~nm}$ for B-, G-, and R-QDs, respectively. The output PL intensity data of the QDs from the deconvoluted spectra were plotted as a function of the propagation distance on a logarithmic scale (Figure $3 \mathrm{~d}$ ). The $\alpha$ values were also calculated. When the propagation distance increased, the PL intensity of the QDs initially increased due to the capillary effect (more concentrated QDs) at the boundaries of the QDs, and then exponentially decreased. The decay constants were estimated to be

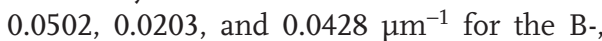
G-, and R-QDs, respectively. The measured decay constants for emissions from the QDs are in reasonable agreement with the finite difference time domain (FDTD) calculation values of $0.1036,0.0670$, and $0.0595 \mu^{-1}$ for 
(a)

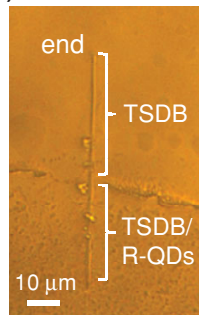

(b)

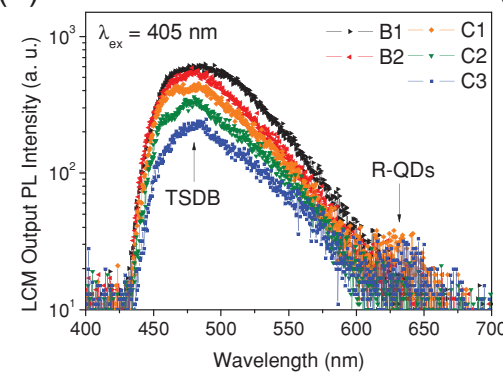

(c)

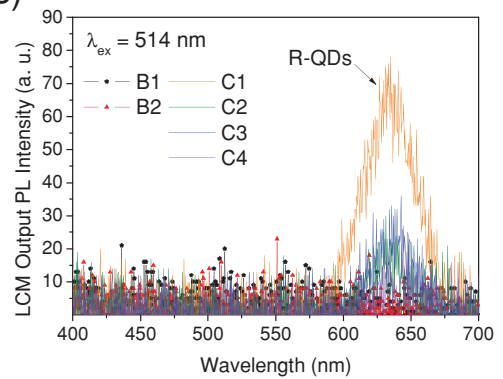

Figure 4. (a) A microscope image of a TSDB/R-QD hybrid NW. Output LCM PL spectra of the TSDB/R-QD hybrid NW with laser excitation wavelengths of (b) $\lambda_{\mathrm{ex}}=405 \mathrm{~nm}$ and (c) $\lambda_{\mathrm{ex}}=514 \mathrm{~nm}$.

the emissions from B-, G-, and R-QDs, respectively. The main factor that limited the propagation of the emission from B-QDs was higher optical extinction in the shorter wavelengths region due to the re-absorption of the propagating light in the TSDB NWs. For longer wavelength emissions of QDs, the re-absorption is minimal. Overall, as shown in Figure S4, the main loss was contributed by the radiative coupling to the substrate. This could be reduced by using a different substrate, such as an air gap in the PDMS microchannel stamp (see Figure 2).

To further investigate the optical waveguiding properties of QDs in a TSDB NW, we used two different excitation sources ( $\lambda_{\mathrm{ex}}=405 \mathrm{~nm}$ and $514 \mathrm{~nm}$ ). Figure 4a shows an optical image of a TSDB NW ( $n=1.71$ at $\lambda=488 \mathrm{~nm}$ and $n=1.77$ at $\lambda=630 \mathrm{~nm})$ hybridized with R-QDs, for which half of the NW's surface was coated with R-QDs. Excitation with a $405 \mathrm{~nm}$ laser yielded two output PL peaks (C1-C3) at $488 \mathrm{~nm}$ and $635 \mathrm{~nm}$, attributable to the TSDB NW and R-QDs, respectively (Figure 4b). When an Ar-He laser $\left(\lambda_{\text {ex }}=514 \mathrm{~nm}\right)$ was used for excitation only the R-QD emission at $635 \mathrm{~nm}(\mathrm{C} 2, \mathrm{C} 3$, and C4) was observed and the output PL of TSDB NW was missing (Figure 4c). This is because the energy of $514 \mathrm{~nm}$ excitation was not sufficiently high to excite the TSDB NW to generate fluorescence. The results imply that the desired color of a waveguided light passing through a given TSDB NW can be tuned using various external excitation wavelengths. The QDs studied here can also be used as nanoscale blue, green, and red light sources for the optical waveguide, and the crystalline TSDB NW can serve as an active fluorescent connector/optical waveguiding fiber in nanophotonic circuits.

\subsection{White Light Optical Waveguide Using TSDB/QD Hybrid NWs}

To investigate the properties of a possible white light optical waveguide, the blue light-emitting TSDB NWs $(n=1.71$ at $\lambda=488 \mathrm{~nm}, n=1.74$ at $\lambda=532 \mathrm{~nm}$, and $n=1.77$ at $\lambda=$ $630 \mathrm{~nm}$ ) were hybridized with green and red light-emitting QDs. Figure 5a shows a color CCD image of the output signal obtained from TSDB NWs coated with G- and R-QDs. Figure $5 \mathrm{a}$ shows a relatively strong blue emission from the TSDB NWs along with the red and green light output signals from the QDs. According to the analyzed optical waveguide characteristics of each color passing through the TSDB NWs, an optimum excitation point can be determined that yields white PL emission. As shown in Figure 5b, broad output emission spectrum was clearly observed with three distinguishable PL spectra centered at 478, 523, and $638 \mathrm{~nm}$, which originated from the TSDB NW, G-QDs, and R-QDs, respectively. For the reference pristine TSDB NW without QDs, the output PL peak was observed at $488 \mathrm{~nm}$, as shown in the inset of Figure 5b. The decay constants were estimated to be $0.0227 \mu^{-1}$ for the TSDB NW $\left(\lambda_{\mathrm{em}}=488 \mathrm{~nm}\right), 0.0203 \mu \mathrm{m}^{-1}$ for the G-QDs $\left(\lambda_{\mathrm{em}}\right.$ $=532 \mathrm{~nm})$, and $0.0428 \mu \mathrm{m}^{-1}$ for the R-QDs $\left(\lambda_{\mathrm{em}}=638 \mathrm{~nm}\right)$. Because each color in the waveguided light has its own propagation efficiency, the composition of polychromatic light can be determined on the basis of the excitation point on the NW. The CIE coordinates were determined to be $(0.281,0.359)$, thus indicating that the light is near the white range. The results show that optical signals with different decay characteristics could be transported through the crystalline organic NW com- (a)

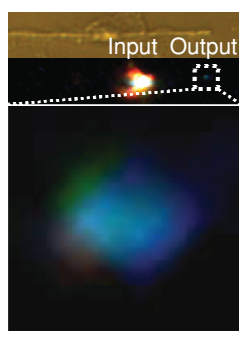

(b)

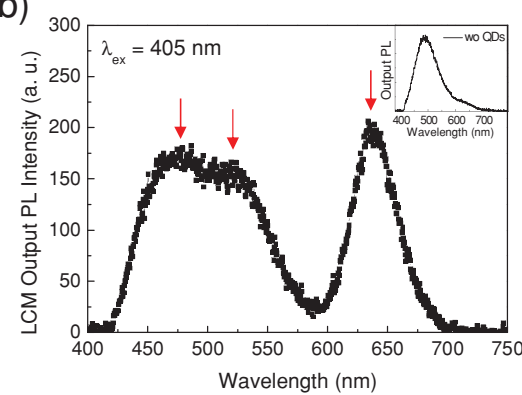

(c)

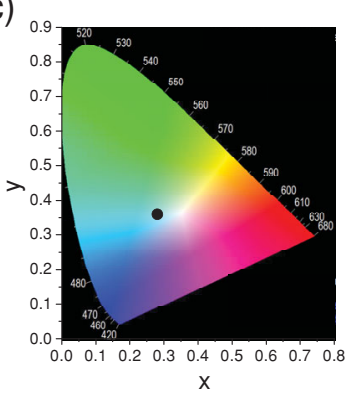

Figure 5. (a) A microscope image (top) and a color CCD image (bottom) of the waveguided output signal from a TSDB/G-QD/R-QD hybrid NW. (b) LCM PL spectrum of waveguided emissions from the TSDB/G-QD/R-QD hybrid NW. Inset: LCM PL spectrum at the end of a hybrid TSDB NW without QDs. (c) CIE coordinate $(0.281,0.359)$ from near-white light waveguided emissions of the TSDB/G-QD/R-QD hybrid NW. 
posed of $\pi$-conjugated TSDB molecules. The presented organic waveguide design can be further devised for efficient transporting of optical information in the fields of quantum information and nanophotonics.

\subsection{Remote Biosensing Using TSDB/G-QD Hybrid NW}

We have observed the efficient waveguiding of various QDs emissions through the $\pi$-conjugated TSDB NW, even though some light scattering existed at the interface. To demonstrate the applicability of this system to biological sensors, we designed the remote biosensing experiment in which the waveguided PL signals were used for detection of Cy3 (fluorescence dye) labeled oligonucleotides attached to the end of the NW. The Cy3 fluorescent dye labeled oligonucleotides (the sequence that was used here was 6-mer anthrax lethal factor $\left(\mathrm{NH}_{2}-5^{\prime}\right.$-GTG TGT-3')) were prepared in distilled water $(1 \mathrm{mM})$. The Cy3 labeled oligonucleotide molecules have main and shoulder absorption peaks at about 550 and $510 \mathrm{~nm}$, respectively. ${ }^{[17]}$ The spectral overlap between G-QDs (or TSDB) and Cy3s (target dye) is sufficient to induce the Förster resonance energy transfer (FRET) effect and/or light absorption, particularly for G-QDs $\left(\lambda_{\mathrm{em}}=535 \mathrm{~nm}\right)$. The optical waveguided remote bio-sensing experiments for detection of Cy3-labeled oligonucleotides were performed by using the pristine TSDB NW $(n$ $=1.71$ at $\lambda=488 \mathrm{~nm}, n=1.74$ at $\lambda=532 \mathrm{~nm}$, and $n=1.77$ at $\lambda=630 \mathrm{~nm}$ ) and the TSDB/G-QDs hybrid NW, as shown in Figure $6 \mathrm{a}$ and $\mathrm{b}$, respectively. The biomaterials were partially coated on the right end of the NW. When the excitation laser irradiated the middle of the pristine TSDB NW, two distinct PL peaks of the waveguided PL spectrum at $480 \mathrm{~nm}$ (due to TSDB $\mathrm{NW}$ ) and $580 \mathrm{~nm}$ (due to Cy3 fluorescent dye) were observed (Figure 6c), and these peaks were associated with the partially covered biomaterials on the right end of the TSDB NW. The inset of Figure $6 \mathrm{c}$ shows the PL spectrum obtained from the excitation position without Cy3 dyes. The detection of the Cy3 fluorescent dye using the waveguided TSDB PL signal could be considered as a realization of remote biosensing.

As shown in Figure 6b, we used the same TSDB NW for hybridization of QDs. The G-QDs were attached to the middle of the TSDB NW and their waveguided emissions were also observed in CCD image (Figure 6b). The waveguided PL peaks at $497 \mathrm{~nm}$ (due to TSDB NW) and $580 \mathrm{~nm}$ (due to Cy3 fluorescent dye) were simultaneously observed at the right end of the NW (Figure 6d). The waveguided PL peak in the blue light region (due to TSDB NW) was somewhat smaller in magnitude mainly due to the scattering loss by G-QDs attached to the NW. However, the output PL intensity at $580 \mathrm{~nm}$ (due to Cy3) considerably increased compared with the intensity obtained using the pristine TSDB NW (Figure 6c), on the same PL intensity scale. This result indicates that G-QD emission originated from the laser excitation location induced (together with the TSDB NW emission) the fluorescence of Cy3 attached to the right end of the NW. There was also a distinct shoulder PL peak corresponding to the green light emitted from G-QDs. With the hybridization of G-QDs on the TSDB NW, there was nearly three-fold increase in PL intensity for the Cy3 at $580 \mathrm{~nm}$ indicating emission from G-QDs contributed to the excitation of the Cy3 fluorescence. Even

(a)

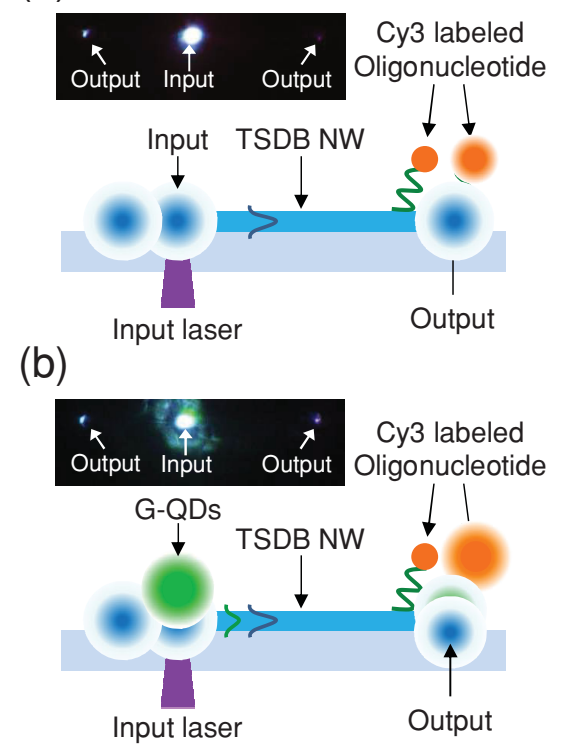

(c)

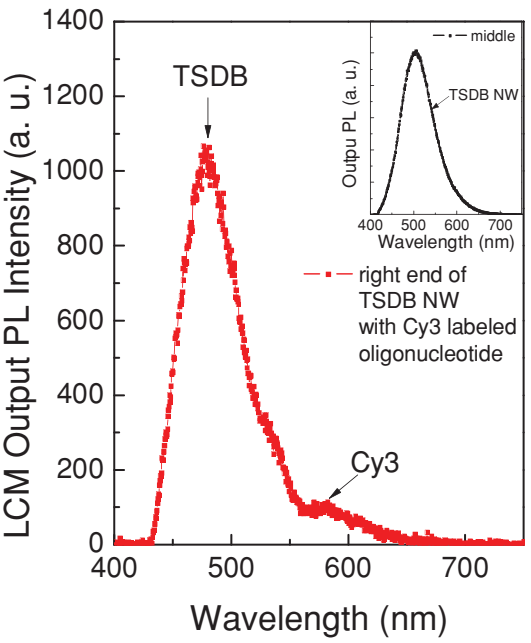

(d)

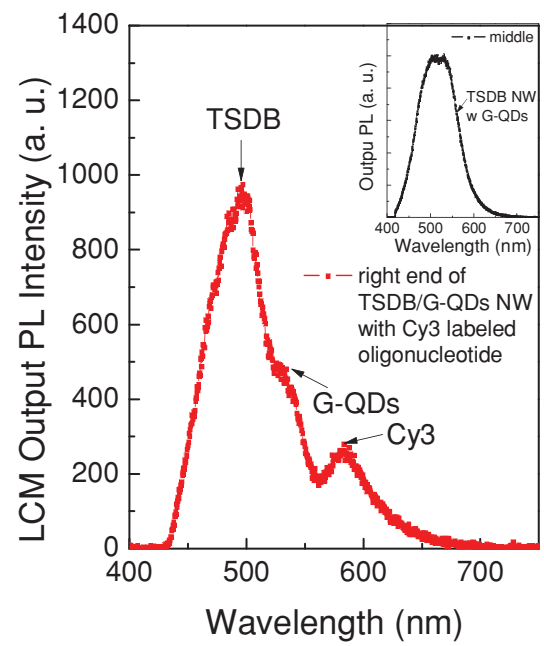

Figure 6. (a) Schematic illustration of the waveguiding experiment for remote biosensing using the TSDB NW. Cy3 labeled oligonucleotides were attached to the right end of the NW (bottom). A color CCD image of the waveguided emission in the corresponding experiment (top). (b) Schematic illustration of the waveguiding experiment for remote biosensing using the TSDB/G-QD hybrid NW. Cy3 labeled oligonucleotides were attached to the right end of the TSDB NW, and G-QDs were attached to the excitation position (bottom). A color CCD image of the waveguided emission in the corresponding experiment (top). (c) Output LCM PL spectrum of the waveguided emission of the TSDB NW with biomaterials (oligonucleotides). Inset: LCM $\mathrm{PL}$ spectrum at the excitation position (middle of the pristine TSDB NW). (d) Output LCM PL spectrum of the waveguided emission of the TSDB/G-QD hybrid NW with biomaterials (oligonucleotides). Inset: LCM PL spectrum at the excitation position (middle of the hybrid TSDB/G-QD NW). 
though the coating of QDs on the NW might lead to light scattering, the results indicate the efficient coupling of light emission from QDs into the NW and sufficiently high output PL intensity for use in sensing applications, manifested in the enhancement of remote bio-sensing efficiency for detection of Cy3 labeled oligonucleotides.

\subsection{Discussion}

The observed efficient polychromatic waveguiding properties of TSDB NWs are attributed to advantageous properties of TSDB molecules. TSDB is a blue-light emitting material and is almost transparent above the wavelength of $\sim 600 \mathrm{~nm}$, providing lossless optical propagation in some of the visible and near IR wavelengths range. The main reason for the observed finite propagation at the wavelengths of $532 \mathrm{~nm}$ and $633 \mathrm{~nm}$ was the radiation loss to the air and to the substrate. Scattering off the QDs attached to a TSDB NW could also contribute to the propagation loss. However, the efficiency with which the QDs emission was optically waveguided through the NW was satisfactory for remote bio-sensing (Figure 6). The large refractive index of TSDB NWs (1.71-1.77) results in a smaller cut-off waveguide's width compared with other organics, which is preferable for achieving nanosize optical waveguide. High refractive index of TSDB also provides better confinement of propagating mode and thus lowers the radiation loss to the substrate. By using finite difference time domain (FDTD), we designed another NW, having a refractive index of 1.6, and found that most of the light was not confined to the NW and escaped through the glass substrate after $\sim 2 \mu \mathrm{m}$ from the launching location, increasing the decay constant of the $635 \mathrm{~nm}$ wavelength about ten times, from $0.0595 \mu \mathrm{m}^{-1}$ to $0.417 \mu \mathrm{m}^{-1}$. This clearly demonstrates the advantages of TSDB's high refractive index in efficient light waveguiding.

A self-assembled TSDB NW is an active fluorescence optical waveguiding system, with optoelectrical properties that differ from those of insulating polystylene (PS) and polymethyl methacrylate (PMMA) nano- or micro-wires reported as polymer optical waveguiding systems. ${ }^{[13]}$ For example, an optical signal can be transported through the semiconductor TSDB NW by the mechanism of the propagation of exciton-polariton $(\mathrm{EP})^{[9]}$ as well as scattering (reflection and refraction). The $\pi$-conjugate structure of TSDB molecules and their intermolecular interaction can be important for the EP characteristics. Previous reports described observations of multiple color waveguidied luminescence and asymmetric light propagation using the composition-graded inorganic semiconducting $\mathrm{NWs}^{[18]}$ as well as the rectified waveguiding phenomenon observed using the organic segmented (i.e., serial heterojunction structure) microwires. ${ }^{[19]}$ However, the fabrication of TSDB/QD hybrid NWs for polychromic optical waveguiding reported here was relatively simple and the systems can be easily modified for various photonic applications through the various hybrid methods of QDs as a post treatment.

\section{Conclusion}

Polychromatic optical waveguiding was successfully demonstrated using a blue light-emitting organic crystalline TSDB NW hybridized with the red, green, and blue light-emitting $\mathrm{CdSe} / \mathrm{ZnS}$ QDs. We observed distinct decay characteristics of the emission from the red, green, and blue light-emitting QDs in TSDB NWs. The composition of polychromatic waveguided light could be controlled by changing the propagation distance. Hybrid NWs of TSDB/G-QDs/R-QDs have shown a near white-color waveguiding with three constituent wavelengths centered at 488, 532, and $638 \mathrm{~nm}$. These peaks cover the visible range, and the proportion of each color's component was calculated in terms of the CIE coordinates. We believe that the light emission from QDs propagated to TSDB NWs and then was optically waveguided through $\pi$-conjugated TSDB molecules. The efficient waveguiding property of the hybrid NW system can be attributed to a strong coupling between QD excitons and propagated polaritons in the TSDB NW. We have demonstrated that the wavelengths in the entire visible range (red, green, and blue) can propagate through $\pi$-conjugated organic crystalline TSDB NWs, and that the output color could be tuned using hybridization, with different combinations of light-emitting QDs. We also observed the enhancement of the PL intensity for remote detection of Cy3 dye attached bio-materials by means of the waveguided QDs emissions and the efficient coupling of the QDs emission to the NW.

\section{Experimental Section}

Synthesis of TSDB: All starting materials were purchased from commercial suppliers (Sigma Aldrich and Fisher Scientific). Synthetic procedures are summarized in Scheme 1, and the synthesized compounds were fully characterized by ${ }^{1} \mathrm{H}-\mathrm{NMR}$ (nuclear magnetic resonance) and GC-mass (gas chromatography). The 1,1'-[(2,5-dibromo1,4-phenylene)bis(methylene)]bis [1,1,1-triphenyl-phosphonium dibromide (1, see Scheme 1) was prepared as previously described. ${ }^{20]}$ The 1,1'-[(2,5-dibromo-1,4-phenylene) bis (methylene)]bis[1,1,1-triphenylphosphonium dibromide (1 g, $1.057 \mathrm{mmol}$ ) and 3,5-bis(trifluoromethyl)

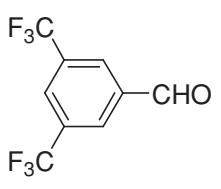<smiles>P=Pc1cc(Br)c(P)cc1Br</smiles>

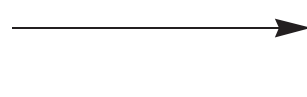

1

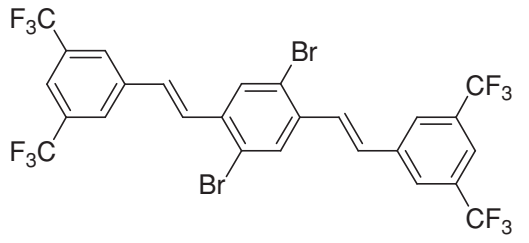

TSDB

Scheme 1. Schematic synthesis procedure for the TSDB molecule. 
benzaldehyde $(0.51 \mathrm{~g}, 2.114 \mathrm{mmol})$ were dissolved in anhydrous tetrahydrofuran. Potassium tert-butoxide $(0.3 \mathrm{~g}, 2.5 \mathrm{eq})$ was proportionally added over the duration of $10 \mathrm{~min}$. Then, the mixture was warmed to reach room temperature and was stirred overnight. After pouring the mixture into water, the precipitate was collected and washed with methanol. Finally, the TSDB powder was obtained through recrystallization from a chloroform solution after flash column chromatography with a chloroform eluent. The TSDB powder dissolved in chloroform was filtered using a syringe filter. This solution was dried on a cover glass at room temperature. Then, the TSDB molecules in the supersaturated solution were self-assembled to the NWs within a few minutes. ${ }^{1} \mathrm{H}-\mathrm{NMR}\left(400 \mathrm{MHz}, \mathrm{CDCl}_{3}\right) \delta 7.92(\mathrm{~s}, 4 \mathrm{H}), 7.88(\mathrm{~s}, 2 \mathrm{H}), 7.78$ (s, 2H), 7.47 (d, 2H), 7.09 (d, 2H), and m/z EIMS 712.

Synthesis of CdSe/ZnS QDs: The CdSe (core) and ZnS (shell) QDs were prepared using a single-step synthesis with a ligand exchange. ${ }^{[21]}$ The surface of the CdSe/ZnS core-shell QDs was functionalized with oleic acid (OA), which acts as a stabilizing ligand during the formation of $\mathrm{CdSe} / \mathrm{ZnS}$ QDs. The OA was subsequently replaced with a stabilizing 11-mercapto-1-undecanol ligand via a reaction with undecanethiol.[22] The 11-mercapto-1-undecanol functionalized $\mathrm{CdSe} / \mathrm{ZnS}$ QDs with $\mathrm{OH}$ groups were easily attached to the surfaces of TSDB NWs. The half surface of a TSDB NW on a cover glass was arranged with QDs solutions ( $5 \mathrm{mg} / \mathrm{mL}$ in methanol solvent) using a micro-pipette (Eppendorf, Manual Pipetting 1-ch: Research) and dried at room temperature. In this study, the minimum axial length covered with the QDs on the NW was about $25 \mu \mathrm{m}$ using the micro-pipette. For future study, the precise control of the QDs' area on the NW can be possible through the use of a nano-pipette.

Measurements: The UV-vis absorption spectra of TSDB NWs and QDs dispersed in methanol were measured using a HP8453 spectrometer at room temperature. For the LCM PL experiments, an unpolarized diode laser $\left(\lambda_{\text {ex }}=405 \mathrm{~nm}\right)$ was used for PL excitation. The spot size of the focused laser beam on the sample was estimated to be $200 \mathrm{~nm}$. The laser's power and the acquisition time incident on the sample for the LCM PL spectra were fixed at $10 \mu \mathrm{W}$ and $20 \mathrm{~ms}$, respectively. The detection position of the waveguided light, at which the output signal was measured, was fixed at the end of a NW. Using the LCM system, both the excitation and the detection points were placed normally to the NW. In addition, the input laser could be moved along the axial direction of the NW. To measure the waveguiding properties of suspended NWs, the patterned PDMS stamps (channel length was several millimeters and channel's cross-section was $25 \mu \mathrm{m} \times 10 \mu \mathrm{m}$ ) were prepared on the cover glass using a standard lithography and TSDB NWs were drop casted on the patterned stamp and one of the suspended NWs was selected for the experiment. The Cy3 fluorescent dye labeled oligonucleotides was purchased from Bioneer. For the exciton lifetime measurements, a timeresolved fluorescence confocal microscope (Micro Time-200, PicoQuant GN003) with a diode laser $\left(\lambda_{\mathrm{ex}}=375 \mathrm{~nm}\right)$ and filter centered at $630 \mathrm{~nm}$ for the red QDs were used. Then, the resulting emissions were collected by time-correlated single photon counting (TCSPC). The time resolution of TCSPC and the instrument response function's full width at half maximum were 4 ps and 240 ps, respectively.

\section{Supporting Information}

Supporting Information is available from the Wiley Online Library or from the author.

\section{Acknowledgements}

This work was supported by the National Research Foundation (NRF) of Korea grant, funded by the Ministry of Science, ICT \& Future Planning (MSIP) (No. 2012R1A2A2A01045102). The TJ Park doctoral
Fellowship, funded by the POSCO TJ Park Foundation, also supported this work. K.-S. Lee acknowledges the funding received for this work from the Asian Office of Aerospace Research and Development (AOARD FA 2386-11-1-4043). Jinsang Kim acknowledges WCU (World Class University) program through the NRF of Korea, funded by MSIP (R31. 2008-000-10075-0) for the postdoctoral fellowship to D.H. Park and the Converging Research Center Program funded by the Ministry of Science, ICT and Future Planning (Project No. 2013K000314). Jeongyong Kim acknowledges the financial support received from the Institute for Basic Science (IBS) (EM 1304) in Korea.

Received: December 2, 2013 Revised: January 13, 2014 Published online: February 27, 2014

[1] M. Law, D. J. Sirbuly, J. C. Johnson, J. Goldberger, R. J. Saykally, P. Yang, Science 2004, 305, 1269

[2] C. J. Barrelet, A. B. Greytak, C. M. Lieber, Nano Lett. 2004, 4, 1981.

[3] W. L. Barnes, A. Dereux, T. W. Ebbesen, Nature 2003, 424, 824.

[4] R. Yan, P. Pausauskie, J. Huang, P. Yang, Proc. Natl. Acad. Sci. USA 2009, 106, 21045

[5] D. O'Carroll, I. Lieberwirth, G. Redmond, Nat. Nanotechnol. 2007 2, 180.

[6] a) F. Balzer, V. G. Bordo, A. C. Simonsen, H.-G. Rubahn, Appl. Phys. Lett. 2003, 82, 10; b) K. Takazawa, Y. Kitahama, Y. Kimura, G. Kido, Nano Lett. 2005, 5, 1293; c) Y. Che, X. Yang, K. Balakrishnan, J. Zuo, L. Zang, Chem. Mater. 2009, 21, 2930; d) Y. S. Zhao, A. Peng, H. Fu, Y. Ma, J. Yao, Adv. Mater. 2008, 20, 1661; e) Y. S. Zhao, J. Xu, A. Peng, H. Fu, Y. Ma, L. Jiang, J. Yao, Angew. Chem. Int. Ed. 2008, 120, 7411; f) Y. S. Zhao, P. Zhan, J. Kim, C. Sun, J. Huang, ACS Nano 2010, 4, 1630.

[7] S. Lal, S. Link, N. J. Halas, Nat. Photon. 2007, 1, 641.

[8] R. Yan, D. Gargas, P. Yang, Nat. Photon. 2009, 3, 569.

[9] K. Takazawa, J.-i. Inoue, K. Mitsuishi, T. Takamasu, Phys. Rev. Lett. 2010, 105, 067401

[10] D. G. Lidzey, D. D. C. Bradley, M. S. Skolnick, T. Virgili, S. Walker, D. M. Whittaker, Nature 1998, 395, 53.

[11] A. L. Briseno, S. C. B. Mannsfeld, X. Lu, Y. Xiong, S. A. Jenekhe, Z. Bao, Y. Xia, Nano Lett. 2007, 7, 668

[12] I. McCulloch, H. Yoon, J. Polym. Sci. Part A: Polym. Chem. 1995, 33, 1177.

[13] F. Gu, L. Zhang, X. Yin, L. Tong, Nano Lett. 2008, 8, 2757.

[14] J. W. Lee, K. Kim, J. S. Jung, S. G. Jo, H.-m. Kim, H. S. Lee, J. Kim, J. Joo, Org. Electron. 2012, 13, 2047.

[15] Y. S. Zhao, P. Zhan, J. Kim, C. Sun, J. Huang, ACS Nano 2010, 4 1630.

[16] F. Balzer, V. G. Bordo, A. C. Simonsen, H. G. Rubahn, Phys. Rev. B 2003, 67, 115408

[17] C. Cui, D. H. Park, J. Kim, J. Joo, D. J. Ahn, Chem. Commun. 2013, 49,5360

[18] a) J. Xu, X. Zhuang, P. Guo, W. Huang, W. Hu, Q. Zhang, Q. Wan, X. Zhu, Z. Yang, L. Tong, X. Duan, A. Pan, Sci. Rep. 2012, 2, 820 b) J. Xu, X. Zhuang, P. Guo, Q. Zhang, W. Huang, Q. Wan, W. Hu, X. Wang, X. Zhu, C. Fan, Z. Yang, L. Tong, X. Duan, A. Pan, Nano Lett. 2012, 12, 5003.

[19] a) Y. Lei, Q. Liao, H. Fu, J. Yao, J. Am. Chem. Soc. 2010, 132, 1742 b) C. Zhang, Y. Yan, J. Yao, Y. S. Zhao, Adv. Mater. 2013, 25, 2854

[20] J. E. Kim, S. Y. Song, H. K. Shim, Synth. Met. 2001, 121, 1665.

[21] W. K. Bae, K. Char, H. Hur, S. Lee, Chem. Mater. 2008, 20, 531

[22] J.-J. Park, P. Prabhakaran, K. K. Jang, Y. Lee, J. Lee, K. Lee, J. Hur, J.-M. Kim, N. Cho, Y. Son, D.-Y. Yang, K.-S. Lee, Nano Lett. 2010, 10, 2310. 